IEEE Trans Ultrason Ferroelectr Freq Control. Author manuscript; available in PMC 2014 March 31.

Published in final edited form as:

IEEE Trans Ultrason Ferroelectr Freq Control. 2013 September ; 60(9): 1853-1867. doi:10.1109/TUFFC.

\title{
S $^{2} d^{2} l^{27} s^{1} f-k$ migration for plane wave ultrasound imaging
}

\author{
Damien Garcia ${ }^{1,2,3}$, Louis Le Tarnec ${ }^{1,2}$, Stéphan Muth ${ }^{1,2}$, Emmanuel Montagnon ${ }^{2,4}$, \\ Jonathan Porée ${ }^{2,4}$, and Guy Cloutier $2,3,4$ \\ ${ }^{1}$ RUBIC, Research Unit of Biomechanics and Imaging in Cardiology \\ ${ }^{2} \mathrm{CRCHUM}$, Research Center, University of Montreal Hospital, Canada \\ ${ }^{3}$ Department of Radiology, Radio-Oncology and Nuclear Medicine, and Institute of Biomedical \\ Engineering, University of Montreal, Canada \\ ${ }^{4}$ LBUM, Laboratory of Biorheology and Medical Ultrasonics
}

\section{Abstract}

Ultrafast ultrasound is an emerging modality that offers new perspectives and opportunities in medical imaging. Plane wave imaging (PWI) allows one to attain very high frame rates by transmission of planar ultrasound wavefronts. As a plane wave reaches a given scatterer, the latter becomes a secondary source emitting upward spherical waves and creating a diffraction hyperbola in the received RF (radio-frequency) signals. To produce an image of the scatterers, all the hyperbolas must be migrated back to their apexes. In order to perform beamforming of plane wave echo RFs and return high-quality images at high frame rates, we propose a new migration method carried out in the frequency-wavenumber $(f-k)$ domain.

The $f-k$ migration for PWI has been adapted from the Stolt migration for seismic imaging. This migration technique is based on the exploding reflector model (ERM), which consists in assuming that all the scatterers explode in concert and become acoustic sources. The classical ERM model, however, is not appropriate for PWI. We showed that the ERM can be made suitable for PWI by a spatial transformation of the hyperbolic traces present in the RF data. In vitro experiments were performed to sketch the advantages of PWI with Stolt's $f$ - $k$ migration over the conventional delayand-sum (DAS) approach. The Stolt's $f$ - $k$ migration was also compared with the Fourier-based method developed by J-Y Lu.

Our findings show that multi-angle compounded $f-k$ migrated images are of quality similar to those obtained with a state-of-the-art dynamic focusing mode. This remained true even with a very small number of steering angles thus ensuring a highly competitive frame rate. In addition, the new FFT-based $f-k$ migration provides comparable or better contrast-to-noise ratio and lateral resolution than the Lu's and DAS migration schemes. Matlab codes of the Stolt's $f-k$ migration for PWI are provided. 


\section{Introduction}

Conventional medical ultrasound imaging consists in scanning a medium using a series of successive focused or multi-focused beams sweeping along the region of interest. The resulting scanlines are then stacked together to reconstruct a single image. The time required to build one frame is thus proportional to the number of gathered lines and the maximal imaging depth. Technological advances within the last decade (parallel computing, high performance data transfer, high-speed processors...) now allows producing ultrasound scanners able to generate a full image from a single transmit thus offering the opportunity of ultrafast imaging. One method to get ultrafast images is by means of plane wave insonifications [1]: a planar wavefront is generated by exciting the transducer elements equally and the radiofrequency (RF) echoes are acquired simultaneously by each element to reconstruct an image in post-processing. Whereas focusing approaches concentrate the acoustic energy at one or several locations, plane wave generation tends to minimize the differences in amplitude and phase over the cross-section delimited by the wavefront. Contrarily to conventional ultrasound imaging, the frame rate reached by plane wave ultrasonography is only limited by the time required for a wave to make a two-way travel. Theoretically, frame rates up to $15,000 \mathrm{~Hz}$ can thus be attained for a 5-cm imaging depth [2]. Recent studies indicate the growing emergence of ultrasound plane wave imaging (PWI). This technique has proven itself as a reliable method in several original and promising applications such as transient elastography, ultrafast Doppler imaging, ultrafast vector flow mapping, electromechanical wave imaging and functional brain imaging [3-7].

As the downward plane wave reaches a single point scatterer, the latter becomes a secondary source emitting upward waves (Fig. 1). Assuming a constant speed of sound within the medium, this source gives rise to a hyperbolic travel-time curve whose eccentricity is governed by the scatterer depth (Fig. 1): the deeper the scatterer, the flatter the hyperbola. More generally, each point scatterer within the insonified medium generates a diffraction hyperbola. The hyperbola thus represents the inhomogeneous point spread function for PWI. In order to produce an image of the scatterers, these diffraction hyperbolas need to be coalesced back into their apexes. In this manuscript, by analogy with seismic imaging, this beamforming process is called migration.

Migration improves focusing by essentially achieving amplitude and phase rectifications to correct for the effects of the spreading of ray paths as waves propagate [8]. In its simplest form, migration by summation of trace amplitudes along hyperbolic trajectories (known as "diffraction summation") has been a basic tool for geophysicists since the 1950s [9]. This method has been extensively used in ultrasound imaging under the name of delay-and-sum (DAS). Adapting this approach to the context of PWI, Montaldo et al. thoroughly described a migration technique based on the two-way travel times for an acoustic wave to reach a scatterer and get back to the transducer elements [1]. The DAS simply consists in integrating the ultrasound RF signals over all the hyperbolas present in the RF signals. The pixels of the resulting migrated RF image are thus assigned the integral values. To potentially improve the image quality and increase the computational efficiency of PWI migration, we propose a new migration method carried out in the frequency-wavenumber $(f-k)$ domain thus bringing the benefit of much faster computational speed, due to the use of the fast Fourier transform 
(FFT) algorithm, while keeping high contrast-to-noise ratio (CNR) and lateral resolution. The $f-k$ migration for PWI is inspired from the original Fourier migration introduced by Stolt for seismic imaging [10;11]. How the standard Stolt's method is adapted for plane wave insonifications is demonstrated in section 2.

FFT-based beamforming for ultrasonic imaging has been the topic of numerous studies since the early 1980s [12-19]. Most of them are based on the angular spectrum method which consists in decomposing the reflected wavefield into plane waves each propagating at a different angle. Although originally derived for monochromatic waves, the angular spectrum method has been extended to wideband systems [13;14]. Much more computationally efficient FFT-based approaches have been recently inspired from the SAR/SAS (synthetic aperture radar/sonar) community [20-22]. These techniques sometimes referred to as "wavenumber algorithms" solve the focusing problem using one-dimensional interpolations in the Fourier domain [12;17-19]. Though extensively applied for imaging in SAR/SAS, the wavenumber algorithm was originally developed by Stolt for exploration seismology [10]. All these abovementioned studies, however, did not fall within the scope of PWI. Instead, each single element of the transducers must be fired independently, while one or several elements are used in reception, thus somewhat mimicking the ideal zero-offset seismic configuration [10;12]. In order to reach very high frame rates, an FFT-based reconstruction of ultrasound images obtained by plane wave insonifications has been successfully addressed by J-Y Lu and his team [23-25]. This spectral technique has recently been tested by an independent group [26]. In Lu's method, the RF image is essentially remapped in the Fourier domain by interpolating the temporal frequencies. This approach is based on the assumption that the scatterers all behave as monopole sources [27].

In this manuscript, we derive a new Fourier $f-k$ migration technique for plane wave ultrasound imaging by modifying the so-called exploding reflector model. In vitro results are presented to sketch the benefits of the Stolt's $f-k$ migration, in terms of image quality, against dynamic focusing and DAS. The new $f-k$ migration process is also compared with Lu's method. The differences between the three approaches (DAS, $f-k$ migration, Lu's method) are discussed in section 4. A concise and readable Matlab code for the $f-k$ migration is given in the Appendix for the readers familiar with Matlab to grasp the underlying algorithm. In addition, a complete optimized Matlab code with in vitro examples is provided in the online supplementary content and in [28].

\section{Theoretical background: adapting the Fourier domain Stolt's migration for plane wave imaging}

\subsection{The exploding reflector model for plane wave imaging}

The classical seismic Stolt's $f$ - $k$ migration is based on the exploding reflector model (ERM), which consists in assuming that all the reflectors in the medium explode simultaneously and become upward-emitting acoustic sources (Fig. 2) [29]. This postulate reduces the back and forth wave propagation to an upward wave scenario. The $f-k$ migration now aims in timereversing the wave propagation and determining the ERM wavefield at $t=0$ (i.e. at the time of explosion) based on the sole knowledge of the wavefield on the surface $z=0$ (Fig. 2). In 
order for the acoustic wavefield to be properly migrated, the ERM must reflect the actual two-way propagation accurately; in other words, one must seek the exploding sources whose ERM-based hyperbolas fit the actual RF hyperbolas. The $f-k$ migration precisely consists in applying the Stolt method to find these virtual sources, followed by a spatial transformation to recover the actual scatterers' positions. This process is straightforward in the particular zero-offset configuration since one simply needs to halve the wavefield propagation speed (see subsection below). In the context of PWI, however, the classical ERM is not suitable as it stands, but the exploding model turns out to be well adaptable to plane wave insonification, as explained in the next subsections. Let us first mention that there is no delay in reception in the following.

\subsubsection{The simplest case: "one emitter-receiver element” scenario-This} situation is equivalent to the so-called "zero-offset section" encountered in post-stack migration for seismic imaging [30]. Such a zero-offset section is acquired by moving a single coincident emitter/receiver along the recording surface. For ultrasound imaging, by analogy, each element of the transducer both transmits the impulse signals and receives the radiofrequency (RF) echoes, one at a time. As a recent example, this seismic imaging concept was applied for nondestructive industrial evaluation of multi-layered media [19]. In this specific configuration, a scatterer positioned at $\left(x_{S}, z_{S}\right)$ leads to a two-way travel-time (from emitter to scatterer back to receiver) which depends upon the transducer position $x$ and is characterized by the hyperbola given by:

$$
\tau_{s}(x)=\frac{2}{c} \sqrt{\left(x_{s}-x\right)^{2}+z_{s}^{2}}
$$

We use here the notation $\left(x_{S}, Z_{S}\right)$ relative to a linear array: $x_{S}$ stands for the scatterer position parallel to the array and $z_{S}$ represents its depth position relative to the array. The origin $(0,0)$ is located at the center of the first leftmost element. The wave propagation speed $(c)$ is assumed constant in the insonified tissues. The factor 2 in equation (1) reflects the round-trip of the wavefield through the medium. It is now easy to see that an exploding source at ( $\hat{x}_{S}$, $\hat{z}_{S}$ ) within a medium whose propagation speed is $\hat{c}$ - this is a one-way situation (from scatterer to receiver) - yields the following ERM travel-time:

$$
\hat{\tau}_{s}(x)=\frac{1}{\hat{c}} \sqrt{\left(\hat{x}_{s}-x\right)^{2}+\hat{z}_{s}^{2}}
$$

Making equal the travel-times $\tau_{S}$ (Eq. 1) and $\hat{\tau}_{S}$ (Eq. 2) by choosing $\left(\hat{x}_{S}, \hat{z}_{S}\right)=\left(X_{S}, Z_{S}\right)$ and halving the propagation speed (i.e. $\hat{c}=c / 2$ ) makes the ERM and the "one emitter-receiver" scenario alike.

2.1.2. The ERM with a horizontal plane wave-To generate a horizontal plane wave, all the transducer elements must emit synchronously. We thus leave the simple abovementioned scenario. Assuming that a horizontal plane wave is sent within the medium, the travel-time produced by a scatterer positioned at $\left(x_{S}, z_{S}\right)$ is now given by (see [1] for details): 


$$
\tau_{s}(x)=\frac{1}{c}\left(z_{s}+\sqrt{\left(x_{s}-x\right)^{2}+z_{s}^{2}}\right) .
$$

To make the ERM compatible with the horizontal plane wave acquisition, one must fit the hyperbolas given by equations (2) and (3). No simple relation, however, can provide a perfect fitting. Since most of the signal energy is concentrated around the apex of the hyperbola, a compromise is to make equal their apical $0^{\text {th }}$ to $2^{\text {nd }}$ order derivatives. This is done by equalizing $\tau_{S}$ and $\hat{\tau}_{S}$ as well as their first and second derivatives (with respect to $x$ ) at $X=X_{S}\left(=\hat{x}_{S}\right)$, respectively, which yields the following:

$$
\hat{c}=\frac{\sqrt{2}}{2} c \text { and } \hat{z}_{s}=\sqrt{2} z_{s}
$$

In the particular case of a horizontal plane wave, the expressions in (4) make the ERM compatible with PWI. In comparison with the "one emitter-receiver element" scenario, the wavefield propagation speed must not be simply halved. In addition, the depth must be rescaled. These relations make the travel-times given by (2) and (3) almost equal in the vicinity of the apex, and their difference decreases in $O\left(\left(x-x_{S}\right)^{4}\right)$. The phase error $-\Delta \varphi_{S}=$ $2 \pi f_{0}\left|\tau_{S}(x)-\hat{\tau}_{S}(x)\right|$, where $f_{0}$ is the central frequency - related to this approximation in the travel-times is illustrated in Figure 3. One can notice that the phase errors are larger for large obliquities (i.e. for large deviation angles formed by the scatterer with respect to the transducer element). This, however, is expected to have little effects on the migrated images since the reflected acoustic energy is maximal for small obliquity values.

2.1.3. The general case: the ERM with a tilted plane wave-In practice, because the image quality achieved with a single plane wave is suboptimal [1], several slightly tilted consecutive plane wavefronts are transmitted and the backscattered RF signals are coherently compounded. It has been reported that less than 10 angles can provide PWIderived images with similar quality as standard multi-focus methods, thus ensuring a highly competitive frame rate [1]. To be able to perform multi-angle compounding with our method, it must be ensured that the ERM can be adapted to a slant plane wave as well. For a plane wave tilted with an angle $\theta$ from the horizontal (see Fig. 4a), the travel-time becomes (see [1] for details):

$$
\tau_{s}(x)=\frac{1}{c}\left(\sin (\theta) x_{s}+\cos (\theta) z_{s}+\sqrt{\left(x_{s}-x\right)^{2}+z_{s}^{2}}\right) .
$$

As performed in the previous paragraph, one now wants to fit this hyperbola with the ERMbased hyperbola given by equation (2). Again, a perfect fitting cannot be obtained and the strategy is to seek simple transformations that establish the equality of the $0^{\text {th }}$ to $2^{\text {nd }}$ order derivatives. To do so, the RF echoes are first trimmed to eliminate the leading zero signals due to the emission delays (see Fig. 4). The travel-time (5) thus becomes: 


$$
\tau_{s}(x)=\frac{1}{c}\left(\sin (\theta)\left(x_{s}-x\right)+\cos (\theta) z_{s}+\sqrt{\left(x_{s}-x\right)^{2}+z_{s}^{2}}\right) .
$$

Now, let $(a, \beta, \gamma)$ be constant parameters defined by the following equations:

$$
\left\{\begin{array}{c}
\hat{c}=\alpha c \\
\hat{z}_{s}=\beta z_{s} \\
\hat{x}_{s}=x_{s}+\gamma z_{s}
\end{array}\right.
$$

Using the definitions (7), the ERM travel-time given by Eq. (2) becomes:

$$
\hat{\tau}_{s}(x)=\frac{1}{\alpha c} \sqrt{\left(x_{s}+\gamma z_{s}-x\right)^{2}+\beta^{2} z_{s}^{2}} \text {. }
$$

Equalizing $\tau_{S}$ (Eq. 6) and $\hat{\tau}_{S}$ (Eq. 8), as well as their first and second derivatives at $x=x_{S}$, provides the following expressions:

$$
\left\{\begin{array}{c}
\alpha=1 / \sqrt{1+\cos (\theta)+\sin ^{2}(\theta)} \\
\beta=\frac{(1+\cos (\theta))^{\frac{3}{2}}}{1+\cos (\theta)+\sin ^{2}(\theta)} \\
\gamma=\frac{\sin (\theta)}{2-\cos (\theta)}
\end{array}\right.
$$

Note that we get $(\alpha, \beta, \gamma)=\left(\frac{\sqrt{2}}{2}, \sqrt{2}, 0\right)$ when $\theta=0$, as obtained with the horizontal wavefront (Eq. 4). The ERM in conjunction with the transformations defined by (7) and (9) can thus be used to model tilted PWI. The difference between the travel-times given by (6) and (8) decreases in $O\left(\left(x-x_{S}\right)^{3}\right)$.

We have just seen that the ERM can be generalized to model PWI adequately. This is a major point since the ERM is the key element of the $f-k$ migration described below. The whole process is schematized in Figure 4.

\subsection{Stolt's $f-k$ migration}

The migration process aims to reconstruct ultrasound images from the raw RF signals generated in PWI mode. Montaldo et al. recently introduced and successfully tested a delayand-sum (DAS) method defined in the time-space domain [1]. Lu et al. proposed a Fourierbased approach [25]. We now propose a new migration method that also operates entirely in the Fourier frequency-wavenumber domain ( $f-k$ migration).

In the field of seismic imaging, Stolt proposed in 1978 a migration method, commonly called the $f-k$ (or sometimes $\omega-k$ ) migration, allowing the construction of migrated images in the frequency domain [10]. The Stolt $f-k$ migration is currently, by a wide margin, the fastest migration technique but it is limited to a constant propagation wave velocity [11]. 
Although it can be a major drawback in seismology where propagation speeds may vary by a factor of 5 (typically from 1000 to $5000 \mathrm{~m} / \mathrm{s}$, [31]), this constraint is generally less critical in most situations for medical ultrasound where the speed of sound remains around $1500 \mathrm{~m} / \mathrm{s}$ in soft tissues (typically from 1480 to $1670 \mathrm{~m} / \mathrm{s}$, [32]). The $f-k$ migration was developed from the linear wave equation using Fourier transforms. We invite readers to refer to [8] for a well-written and complete description of the Stolt $f-k$ migration. Only the main steps are described here.

Let $\Psi(x, z, t)$ be a scalar wavefield of the ERM satisfying the linear two-dimensional wave equation. One wants to determine the ERM wavefield at the time of explosion, i.e. $\Psi(x, z, t$ $=0)$, knowing the wavefield on the surface $\Psi(x, z,=0, t)$. Now let $\varphi\left(k_{X}, Z, f\right)$ denote the Fourier transform of $\Psi(x, z, t)$ over $(x, t)$, so that:

$$
\Psi(x, z, t)=\iint_{-\infty}^{+\infty} \phi\left(k_{x}, z, f\right) e^{2 i \pi\left(k_{x} x-f t\right)} d k_{x} d f
$$

where $k_{X}$ stands for the spatial wavenumber related to $x$ and $f$ is the temporal frequency. For a constant ERM propagation speed ( $\hat{c}$ ), the application of the Fourier transform to the wave equation yields to the following Helmholtz equation [8]:

$$
\frac{\partial^{2} \phi}{\partial z^{2}}+4 \pi^{2} \hat{k}_{z}^{2} \phi=0
$$

where the wavenumber $\hat{k}_{z}$ is given by:

$$
\hat{k}_{z}^{2}=\frac{f^{2}}{\hat{c}^{2}}-k_{x}^{2}
$$

The unique boundary condition for equation (11) is $\phi\left(k_{X}, 0, f\right)$, which is the Fourier transform of $\Psi(x, z,=0, t)$. To close the ERM migration problem described by equations (11) and (12), it is now assumed that $\Psi(x, z, t)$ contains only waves moving upward (see Eq. 5.48 in [8]). The ERM wavefield is thus allowed to propagate on the $-Z$ direction only as would occur with primary reflections. The wave equation can thus be solved and one obtains the migrated wavefield [8]:

$$
\Psi(x, z, 0)=\iint_{-\infty}^{+\infty} \phi\left(k_{x}, 0, f\right) e^{2 i \pi\left(k_{x} x-\hat{k}_{z} z\right)} d k_{x} d f .
$$

To fully benefit from the Fourier transforms, Stolt proposed to change the variable $\hat{k}_{z}$ by introducing (see equation 12):

$$
f\left(\hat{k}_{z}\right)=\hat{c} \operatorname{sign}\left(\hat{k}_{z}\right) \sqrt{k_{x}^{2}+\hat{k}_{z}^{2}}
$$


This expression describes the spectral remapping of the Stolt's $f-k$ migration for PWI, which differs from the remapping proposed by J-Y Lu [25] (more details are given in section 4.1). Using the change of variables (14), the Stolt's migration solution is finally:

$$
\Psi(x, z, 0)=\iint_{-\infty}^{+\infty} \frac{\hat{c} \hat{k}_{z}}{\sqrt{k_{x}^{2}+\hat{k}_{z}^{2}}} \phi\left(k_{x}, 0, f\left(\hat{k}_{z}\right)\right) e^{2 i \pi\left(k_{x} x-\hat{k}_{z} z\right)} d k_{x} d \hat{k}_{z}
$$

As shown by Eq. (15), the migrated solution is basically the inverse Fourier transform of

$$
\frac{\hat{c} \hat{k}_{z}}{\sqrt{k_{x}^{2}+\hat{k}_{z}^{2}}} \phi\left(k_{x}, 0, f\left(\hat{k}_{z}\right)\right) .
$$

\subsection{The Stolt's $\boldsymbol{f}-\boldsymbol{k}$ migration algorithm for plane-wave ultrasound imaging}

We have just seen how to adapt the exploding reflector model (ERM) for plane-wave ultrasound imaging (PWI) in section 2.1. We also showed that the migrated solution of the ERM is given by equation (15). We now have the framework for the $f-k$ migration of PWIderived RF signals. The three-step process to get $f-k$ migrated images can be summarized as follows:

1. The leading zeros of the RF signals due to the emission delays are first removed (Eq. 6). This is the step $b$ to $c$ in Figure 4. This signal trimming aligns the hyperbolas of same apical curvature horizontally (Fig. $4 c$ ). Note that this operation slightly distorts the hyperbolas (Fig. $4 c$ ), more especially as the steering angle is large.

2. The Stolt's migration algorithm described by Eq. (14) and (15) is applied (step $c$ to $d$ in Fig. 4) using the ERM velocity $\hat{c}$ as defined in Eq. (7) and (9). A scatterer originally located at $\left(x_{S}, z_{S}\right)$ will be moved to $\left(\hat{x}_{S}, \hat{z}_{S}\right)$ after the migration (Fig. $4 d$ )

3. Coordinate transformations $\left(\hat{x}_{S}, \hat{z}_{S}\right) \rightarrow\left(x_{S}, z_{S}\right)$ are performed to get back into the actual position (Fig. $4 d$ ) by using the expressions given by Eq. (7) and (9).

It is worth noting that these basic steps can be implemented in the Fourier domain to gain significant computational speed. A pseudo-code for tilted PWI $f-k$ migration is proposed in Fig. 5 and a simplified Matlab code (for horizontal plane waves only) is given in the Appendix. A complete and optimized Matlab pcode (protected function file) is also provided in the Supplementary materials and in [28]. This latter program was used to generate the $f-k$ migrated images contained in this manuscript.

As illustrated by equations (12) and (15), the Stolt's migration includes a non-uniform Fourier transform since the $\hat{k}_{z}$ 's are not equally sampled. To gain computational speed by using the fast Fourier transform (FFT), the data must be laid on a regular grid by interpolation or other gridding methods, as performed in geosciences or magnetic resonance imaging [33-36]. In our context, the simplest algorithm consisted in linear interpolation after extensive zero-padding in the first FFT: $\Psi(x, Z=0, t) \rightarrow \varphi\left(k_{X}, 0, f\right)$. Although a 
nonuniform FFT [26;35] could have been included in the $f-k$ migration algorithm, we rather used a linear interpolation scheme for the sake of simplicity. As an alternative, the sinc (sine cardinal) interpolation could also be used to reduce the amount of zero-padding and make the algorithm a bit faster [34]. The sinc interpolator option is available in the Matlab pcode given in the Supplementary materials.

\section{In vitro experiments: Stolt's $\boldsymbol{f}-\boldsymbol{k}$ migration against DAS and Lu's method}

As detailed in this section, ultrasound PWI was analyzed in an in vitro phantom to illustrate the performance of the new $f-k$ algorithm for migrating PWI-derived RF data. In vitro plane wave measurements were performed with the Verasonics research scanner (model V-1-128, Verasonics Inc., Redmond, WA). The raw RF signals were transformed using the DAS [1], the $f-k$ migration and the Lu's method [25]. The reconstructed B-mode images were compared with those obtained using state-of-the-art dynamic multi-focusing approaches. The $f-k$ migration and the Lu's method were compared under strictly similar conditions. Only the spectral remapping formulas (see section 4.1 for more details) were different in the algorithms. Other conditions that may affect image quality (interpolation schemes, FFT, zero-padding...) were kept unchanged.

\subsection{Construction of the migrated images}

In vitro $\mathrm{RF}$ data were acquired with the $\mathrm{V}-1$ Verasonics scanner using a $5 \mathrm{MHz}$ linear array transducer (ATL L7-4, 128 elements, pitch $=0.3 \mathrm{~mm}$ ) and the 403GS LE Gammex phantom (Gammex Inc., Middleton, WI). The Gammex phantom was insonified with successive tilted plane waves. No transmit apodization was used. RF raw data backscattered by anechoic mimicking cysts and $0.1 \mathrm{~mm}$ nylon fibers up to $10 \mathrm{~cm}$ deep were acquired at a sampling frequency of $20 \mathrm{MHz}$. The RF signals were zero-phase high-pass filtered to remove any DC offset. The RFs were then migrated using the DAS, $f-k$ and Lu's methods. A $f$-number of 1.75 (i.e. the maximal aperture was given by $z / f=z / 1.75$ ) was used when beamforming with the DAS in order to make the contrast better in the very near field [1]. The migrated RF signals were time-gain compensated and B-mode images were obtained through a Hilbert transform. The B-mode images were gamma-compressed using $\gamma=0.3$ (except for the study of lateral resolution, see subsection 3.4) and converted to 8-bit grayscale. To assess the quality of the images generated by ultrasound plane waves, PWI was compared with the dynamic focusing mode offered by the Verasonics scanner. In the latter approach, 128 dynamically focused scanlines were obtained using seven transmit foci vertically distributed over the region-of-interest, and a $f$-number of 1.75 (as in [1]).

\subsection{Effect of the steering angle on the contrast-to-noise ratio (CNR)}

Multi-angle RF coherent compounding is required with plane wave imaging (PWI) to enhance the quality of the ultrasound images [1]. We first verified the quality of PWIderived B-mode images returned by a single plane wave insonification with a given wavefront angle (defined with respect to the transducer). How the wavefront angle (noted $\theta$ in Eq. 5; see also Fig. 4.a) affects the contrast-to-noise ratio (CNR) of migrated images has been investigated in vitro on a single $6 \mathrm{~mm}$-diameter $3 \mathrm{~cm}$-deep anechoic target. The target CNR in $\mathrm{dB}$ was defined as [37]: 


$$
\mathrm{CNR}=20 \log _{10} \frac{\left|\mu_{\mathrm{t}}-\mu_{\mathrm{b}}\right|}{\sqrt{\left(\sigma_{\mathrm{t}}^{2}+\sigma_{\mathrm{b}}^{2}\right) / 2}}
$$

where $\mu_{\mathrm{t}}$ and $\mu_{\mathrm{b}}\left(\sigma_{\mathrm{t}}^{2}\right.$ and $\left.\sigma_{\mathrm{b}}^{2}\right)$ are the means (variances) of gray levels in the target and the surrounding background, respectively.

The in vitro results show that the CNR decreased when the wavefront angle increased (Fig. 6). The CNR remained almost unchanged with wavefront angles varying from $0^{\circ}$ to $10^{\circ}$ (Fig. 6). Beyond $10^{\circ}$, however, a rapid decrease in CNR was observed with the three migration techniques, which indicates that high wavefront angles (with respect to the probe) should be avoided to obtain high-quality images. In this configuration, no major difference between the three migration methods was noticed below $10^{\circ}$. According to our findings, the absolute wavefront angle for PWI should preferably remain lower than $10^{\circ}$. This observation, however, cannot be generalized since the maximal acceptable steering angle very likely depends upon several factors such as the central frequency and the pitch. As a side note, the contrast, as defined by equation (1) in [37], had a behavior very similar to the contrast-to-noise ratio (see Fig. 6-bis in the online supplementary materials for detailed results).

\subsection{Effect of the number of compounding angles on the contrast-to-noise ratio (CNR)}

The combination of several migrated RF signals acquired with different wavefront angles may significantly improve the quality of the resulting image, more importantly for large depths. Getting one image by coherent compounding was carried out as follows: 1) several RF signals were acquired with slightly different plane wave angles, 2) they were migrated independently then averaged, and 3) an envelope detection was performed in the resulting compounded migrated RF data. To analyze how the number of compounding angles improves the image quality, a series of in vitro tests were performed with anechoic mimicking cysts. The angular range was chosen as $\left[-\theta_{\max } ;+\theta_{\max }\right]$ with an increment of $1^{\circ}$, and $\theta_{\max }$ was varied from $0^{\circ}$ to $10^{\circ}$. For instance, when $\theta_{\max }$ was $3^{\circ}$, seven angles (i.e. $-3^{\circ}$, $-2^{\circ},-1^{\circ}, 0^{\circ}, 1^{\circ}, 2^{\circ}, 3^{\circ}$ ) were used to create a compounded image. The data acquired by PWI were compared with those obtained by the dynamic focusing mode offered by the Verasonics scanner. The CNR of the targets ( 3 and $8 \mathrm{~cm}$-deep anechoic cysts), delimited by dashed circles in Fig. 7, was calculated using equation (17).

As expected, the CNR of the anechoic cysts improved when increasing the number of angles (Fig. 7). This improvement, however, became non-significant above a total number of 9 or 11 angles. The in vitro results show that the three migration methods perform roughly equally although the CNR reached by Lu's method was somewhat lower. Our findings also indicate that compounding PWI with $f$ - $k$ migration using a very few angles (typically 7 to 11) equals the dynamic focusing approach in terms of CNR (10.7 and $9.6 \mathrm{~dB}$ at 3 and $8 \mathrm{~cm}-$ depth, respectively), which ensures a highly competitive frame rate for PWI (only 7 to 11 compounded firing sequences are required) in comparison with the focused imaging (128 $\times$ number-of-foci firing sequences). Similar conclusions were reached with the contrast 
defined by equation (1) in [37] (see Fig. 7-bis in the online supplementary materials for detailed results).

\subsection{Analysis of the lateral resolution}

The effect of the number of compounding angles ( 1 up to 21 angles, with an increment of $1^{\circ}$, as in the previous subsection) on the lateral resolution given by PWI was first analyzed at 3 and $8 \mathrm{~cm}$ depth. Coherent compounding was performed as described in the previous subsection. The lateral resolution slightly improved with the DAS and Lu's approaches when increasing the number of compounding angles, both at 3 and $8 \mathrm{~cm}$ depth (see Fig. 8). The resolution provided by the two Fourier methods ( $f-k$ and Lu's) was better at $8 \mathrm{~cm}$. At 3 $\mathrm{cm}$, the resolution reached with the $f-k$ migration was better with a small number of angles $(0.55 \mathrm{~mm}$ vs. $0.71 \mathrm{~mm})$ and became identical with 21 angles $(\approx 0.60 \mathrm{~mm})$.

The lateral resolution provided by PWI was then compared with that given by the dynamic focusing method with the Verasonics scanner and the 128-element linear array transducer. The effect of the depth on the resolution was assessed in vitro using the $0.1 \mathrm{~mm}$ nylon fibers (up to $10 \mathrm{~cm}$ deep) of the Gammex phantom. Dynamic focusing was obtained using 7 foci and an $f$-number of 1.75 . The lateral resolution was estimated by measuring the full width at half maximum (FWHM) of the real envelopes of the migrated RF signals (no gammacompression was used here).

Seven angles $\left(-3^{\circ}\right.$ to $3^{\circ}$, increment of $\left.1^{\circ}\right)$ were used for the compounded PWI images. The PWI-derived lateral resolution was around $0.6 \mathrm{~mm}(\approx 2$ pitches) until about $4 \mathrm{~cm}$ deep then degraded linearly up to $1.25 \mathrm{~mm}$ ( $\approx 4$ pitches) at $10 \mathrm{~cm}$ (see Fig. 9). The lateral resolution was slightly better with the $f-k$ migration in comparison with the DAS and Lu's method. On average, the lateral resolution achieved by the dynamic focusing mode was better than the $f$ $k$ migrated PWI (difference $=0.17 \pm 0.08 \mathrm{~mm}=0.59 \pm 0.27$ pitch). It is worth noticing, however, that the number of firing sequences was 128 times lower with PWI. The resolution obtained by PWI in our study (between 0.5 and $1.2 \mathrm{~mm}$ ) was somewhat similar to that reported by Montaldo et al. in [1] (around $1.1 \mathrm{~mm}$ at $4.5 \mathrm{MHz}$ with a $0.33 \mathrm{~mm}$ pitch). Counterintuitively enough, however, they did not observe any degradation of the lateral resolution at larger depths. As depth increases, though, the hyperbolas generated by PWI flatten (and become a horizontal line at infinite depth), thus producing some ambiguity in the location of their apexes and consequently reducing the lateral resolution.

\subsection{Computational complexity: Stolt's $\boldsymbol{f}-\boldsymbol{k}$ migration vs. DAS}

To determine the computational complexity of the $f-k$ migration, let $n_{t}$ denote the number of elements in the transducer (in general 64,128 or 192) and $n_{S}$ the number of time samples (typically $\geq 1000-3000$ ). The $f-k$ migration requires two 2-D FFTs and the computation of $\left(n_{t} n_{S}\right)$ interpolated values. In comparison, the DAS proposed by Montaldo et al. must retrieve $n_{t}\left(n_{t} n_{S}\right)$ interpolated data and perform $\left(n_{t} n_{S}\right)$ summations over $n_{t}$ values [1]. The computational complexity of the migrating process thus reduces from $O\left(n_{t} n_{t} n_{s}\right)$ down to $O\left(n_{t} n_{S} \log \left(n_{t} n_{S}\right)\right)$ when performed in the Fourier domain. Note that the Lu's method has the same computational complexity as the $f-k$ migration since only the interpolation scheme is 
different (see subsection 4.1). The number of floating operations is significantly reduced when the migration of the RF data is carried out in the Fourier domain.

Overall, in our study, the $f-k$ migration ran around 25 times faster than the DAS on a CPU (Intel Core i5) operating at $2.80 \mathrm{GHz}$, whatever the number of time samples. However, it is obvious that the actual resources utilized by the algorithms depend upon numerous aspects including the programming language, CPU or GPU speed, available memory, speed of data transfer, serial or parallel transfer, etc. Recent works show that GPU platforms can execute beamforming for plane wave imaging at frame rates well beyond the video display range [38]. In this study, since it was not our goal to get real-time visualization, the RF signals were migrated offline using Matlab.

\section{Discussion}

Ultrafast ultrasound PWI, in comparison with the conventional focusing approaches, allows one to obtain a full image with a single transmit by migration of the resulting RF signals. We have shown that PWI-derived ultrasound images can offer an image quality similar to that provided by a state-of-the-art dynamic focusing approach, but with a frame rate up to 100 times faster. In the present paper, a well-established seismic migration method - the Stolt's $f-k$ migration - has been modified for PWI. Since we are not in the specific zero-offset condition of seismic imaging, we needed to adapt the exploding reflector model (ERM). We demonstrated that the ERM can be made suitable to PWI by fine-tuning the diffraction hyperbolas present in the RF data. The resulting new $f-k$ migration yielded high-quality images in terms of CNR and lateral resolution.

The main advantage of the $f-k$ migration over the conventional DAS is that it totally works in the Fourier space. This makes the algorithm much faster, and any filtering process in the frequency domain can be included without significant increase in computation time. Potential improvements could be further provided to the $f-k$ migration algorithm. As already mentioned, a non-uniform FFT (NUFFT) can be used to get rid of the interpolation process that appears in the Stolt's migration (Fig. 5). As another alternative, since the bandpass RF signals are highly sparse, the $f-k$ migration could be made even faster with the use of sparse Fourier transforms [39;40]. Although the theory has been derived in two dimensions, the $f-k$ migration for PWI can be generalized in three dimensions. It may be expected that threedimensional PWI with a $32 \times 32$ or $64 \times 64$ matrix array would be feasible in the near future. In that case, one would simply have to deal with hyperboloids instead of hyperbolas.

\subsection{Differences between the three migration methods}

In this study, we have shown that the new FFT-based $f-k$ migration provided comparable or better contrast-to-noise ratio and lateral resolution than the Lu's and DAS migration schemes. One must be aware that migration is an ill-posed inverse problem. The wave equation indeed requires two boundary/initial conditions in order to be solved. Available information (acoustic pressure at $\mathrm{z}=0$, only) is insufficient to recover the insonified medium. Additional assumptions are thus required to close the problem. The different migration algorithms precisely differ by the kind of approximations made. 
4.1.1. DAS-The DAS (delay-and-sum) is actually equivalent to the so-called diffraction summation, the simplest migration method used by the geophysicists since the 1960s [9]. This is a geometric strategy which consists in summing the backscattered signals along the hyperbolic traces of the diffraction responses. The DAS thus provides a basic solution of the migration problem. Although this procedure makes good sense and provides good outputs in plane wave imaging, it is theoretically incorrect [29]. A more exact process would be for instance given by the Kirchhoff's integral theorem, which adds amplitude and phase corrections to the data before summation $[29 ; 41 ; 42]$.

\subsubsection{Spectral mapping of the Fourier-based methods-Both Lu and $f-k$}

migrations are Fourier-based methods. The only difference lies in their spectral remapping. Assuming horizontal plane waves only (i.e. no steering angle), one has the ERM speed $\hat{c}=c / \sqrt{2}$ (see Eq. 4). Now choosing $k_{Z}=2 f l c$, i.e. $\hat{k}_{z}=\sqrt{2} f / c$, the spectral remapping for the $f-k$ migration is yielded by equation (14):

$$
f \rightarrow f_{f-k}=\operatorname{sign}(f) \frac{c}{\sqrt{2}} \sqrt{k_{x}^{2}+2 f^{2} / c^{2}} .
$$

Again, assuming no steering angle, Lu's remapping is written as (see Eq. 43 in [25]):

$$
f \rightarrow f_{L u}=f+\frac{c^{2} k_{x}^{2}}{4 f} .
$$

A concise description of the theoretical background leading to this spectral remapping is available in [27]. Using a Taylor series about $\left(k_{X}, f\right)=\left(0, f_{0}\right)$, where $f_{0}$ is the central frequency, it can be shown that the two remapped frequencies are equivalent up to the third order (see the online supplementary materials for more details). Lu's method and $f-k$ migration thus mainly differ for relatively large spatial wavenumbers $k_{X}$. How Lu's method and $f-k$ migration behave in the Fourier domain is illustrated on Fig. 10 (see also Fig. 10.bis in the online supplementary materials). Accordingly, one can notice that their spectra diverge when $\left|k_{X}\right| \gg 0$. On the contrary, the differences between the DAS and Lu's spectra are less significant and more homogeneous (see rightmost panels in Fig. 10).

\subsubsection{Further details regarding Lu's method and Stolt's f-k migration-Lu's} process assumes the scatterers to behave as pure monopole sources: as the plane wave reaches a scatterer, it becomes a source that emits circular waves spreading out uniformly in all directions. In such conditions, the Green's function for the 2-D wave equation is reduced to a Hankel function. When working in the Fourier domain, this leads to an analytical expression linking the received wavefront to the monopole source image. In the real situation, the scatterers behave more realistically as reflectors than monopole sources. The simplest model relating the wavefield to the reflectivity is precisely the exploding reflector model (ERM) as used in the $f-k$ migration [8]. 
The Stolt's $f-k$ migration is based on the ERM and provides an exact solution to the migration problem for constant wave speed [8;10]. In the ERM, exploding sources are located along the reflecting interfaces. These sources explode in concert and emit waves, which propagate upward and are recorded by the receivers at the surface [30]. In comparison with Lu's migration technique, the Stolt migration operates with upward waves only. The ERM model generates normal-incidence reflections. In other words, Stolt's model is basically a reflector model, while Lu's approach is based on a purely scattering model. The ERM, however, works for a zero-offset section; i.e. this model is valid only if each single element of the transducer emits independently (as for standard synthetic aperture, see Introduction). To make the ERM suitable for plane wave imaging, we had to modify the diffraction hyperbolas by using basic spatial transformations (see section 2.1). This numerical step inevitably corrupts the exactness of the Stolt solution. However, because the fitting is performed up to third order in the vicinity of the hyperbola apex and since most of the signal energy is concentrated around the apex of the diffraction hyperbola, the Stolt's $f-k$ migration remains highly accurate in PWI, as confirmed by our in vitro findings.

\subsection{Plane wave imaging vs. dynamic focusing}

An ultrasound image usually contains a hundred of lines. In conventional ultrasound, each scanline is generated by a single- or multi-transmit focused scheme. The state-of-the-art focused technique is "dynamic focusing", which focuses both on transmission and reception [44;45]. In the Verasonics system, receipt focusing is performed by a digital delay-and-sum beamformer and the image is divided into several focal zones. Multi-focus imaging is thus a slow process. Indeed, a total of [number-of-scanlines $\times$ number-of-foci] transmit-receive sequences are required to get a single image. In comparison, only a few steered plane wave insonifications (typically 7 to 11 in this study) provides an image of quality comparable to dynamic focusing (see Fig. 7-9). Of particular importance is the repetitive acoustic energy concentration that occurs with transmit focusing. High acoustic peak pressures are generated by transmit-focusing techniques. In PWI, the acoustic pressure is uniformly spread over the insonified medium. As an example, PWI thus helps to preserve microbubbles and improves ultrasound contrast imaging [46]. To sum up, conventional focus imaging is a high-energy slow modality, while PWI is a low-energy fast process.

\subsection{PWI using $f-k$ migration: limitations and perspectives}

Ultrasound PWI needs the wavefronts to be planar and tilted with the desired incident angle. In order to get high-quality images by PWI, one must ensure that a planar wavefield is synthesized properly. An unlimited number of coplanar elementary sources can produce a perfect slant plane wave. In practice though, the amount of elements in a linear array transducer is limited to 64,128 or 192 . This technical limitation may cause adverse effects that may negatively affect the resulting images. First, the wave equation is not limited to a plane and out-of-plane scatterers may thus contribute to the RF signals. More importantly, a more disturbing effect may rise from the grating lobes which are induced by the regular spacing of the individual transducer elements. It is known that the grating lobes are of larger magnitude as the steering angle increases [47]. This may explain in part why the migration was less efficient beyond $10^{\circ}$. Whether an appropriate transmit apodization can significantly reduce this adverse effect remains to be investigated. Besides the technical aspects, the $f-k$ 
migration model is also subjected to restrictions. This algorithm is based on the 2-D wave equation and the Born approximation, assuming a constant speed of sound and only upcoming waves. In soft tissues, however, the speed of sound is not absolutely uniform [32] and one must therefore use an average or root-mean-square value. As with conventional focusing imaging [48], a mismatch in the speed of sound may significantly affect the quality of the images reconstructed by migration [49]. To illustrate the effect of a speed-of-sound mismatch in the context of plane wave imaging, image quality was determined in the Gammex phantom by repeating the migration processes with different speed values (the speed of sound within the Gammex phantom is claimed to be $1540 \mathrm{~m} / \mathrm{s}$ ). We studied the CNR of the $8 \mathrm{~cm}$-deep cyst as well as the lateral resolution at 3-cm depth (see sections 3.3 and 3.4) for speed of sounds ranged from 1400 to $1700 \mathrm{~m} / \mathrm{s}$ in the algorithms. B-mode images were generated using seven steering angles $\left(-3^{\circ}\right.$ to $\left.+3^{\circ}\right)$. Our measures (Fig. 11) show that image quality indeed quickly degrades when the speed of sound is under- or overestimated. CNR and lateral resolution, however, were little affected in the 1450-1550 (m/s) range.

Several correctors have been proposed to adjust the speed of sound in ultrasound imaging [50-52]. As a side note, because it is a constant speed method, the Stolt's $f-k$ migration would be also limited in transcranial ultrasound imaging since the sound propagates much faster in the skull bone than in the brain. Less limiting models valid for vertically varying velocities, such as the phase-shift method [19;53], would be better adapted in this situation.

\section{Conclusion}

PWI offers the major advantage to yield high-quality images at very high frame rates with low acoustic energy. The Stolt's $f-k$ migration, which is based on a physically sound seismic technique, yields high-quality PWI-derived ultrasound images. An advantage of the $f-k$ migration against the classical DAS is its faster computational speed due to the use of FFTs. More importantly, the $f-k$ migration has the potential to return better lateral resolution. The Stolt's $f-k$ migration has thus the capability to significantly improve ultrasound PWI, and one could bet than PWI can look forward to a prosperous future in medical ultrasound. Further theoretical and experimental investigations will help to identify the best conditions for optimal PWI in different physiological contexts.

\section{Supplementary Material}

Refer to Web version on PubMed Central for supplementary material.

\section{Acknowledgments}

This work was supported by a grant from the Canadian Institutes of Health Research (MOP-106465). Dr. Garcia holds a research scholarship from the Fonds de Recherche en Santé du Québec (FRSQ).

\section{Bibliography}

1. Montaldo G, Tanter M, Bercoff J, Benech N, Fink M. Coherent plane-wave compounding for very high frame rate ultrasonography and transient elastography. IEEE Trans Ultrason Ferroelectr Freq Control. Mar; 2009 56(3):489-506. [PubMed: 19411209] 
2. Bercoff, J. Ultrasound Imaging - Medical Applications. InTech; 2011. Ultrafast Ultrasound Imaging; p. 3-24.

3. Gennisson JL, Deffieux T, Mace E, Montaldo G, Fink M, Tanter M. Viscoelastic and anisotropic mechanical properties of in vivo muscle tissue assessed by supersonic shear imaging. Ultrasound Med Biol. May; 2010 36(5):789-801. [PubMed: 20420970]

4. Bercoff J, Montaldo G, Loupas T, Savery D, Meziere F, Fink M, Tanter M. Ultrafast compound Doppler imaging: providing full blood flow characterization. IEEE Trans Ultrason Ferroelectr Freq Control. Jan; 2011 58(1):134-147. [PubMed: 21244981]

5. Udesen J, Gran F, Hansen K, Jensen JA, Thomsen C, Nielsen MB. High frame-rate blood vector velocity imaging using plane waves: Simulations and preliminary experiments. IEEE Trans Ultrason Ferroelectr Freq Control. Aug; 2008 55(8):1729-1743. [PubMed: 18986917]

6. Provost J, Nguyen VT, Legrand D, Okrasinski S, Costet A, Gambhir A, Garan H, Konofagou EE. Electromechanical wave imaging for arrhythmias. Phys Med Biol. Nov; 2011 56(22):L1-11. [PubMed: 22024555]

7. Mace E, Montaldo G, Cohen I, Baulac M, Fink M, Tanter M. Functional ultrasound imaging of the brain. Nat Methods. Aug; 2011 8(8):662-664. [PubMed: 21725300]

8. Margrave, GF. CREWES Educational Resources. Numerical methods of exploration seismology. 2003. Elementary migration methods; p. 112-188.

9. Hagedoorn JG. A process of seismic reflection interpretation. Geophysical Prospecting. 1954; 2(2): 85-127.

10. Stolt RH. Migration by Fourier transform. Geophysics. 1978; 63(1):23-48.

11. Margrave GF. Direct Fourier migration for vertical velocity variations. Geophysics. 2001; 66(5): 1504-1514.

12. Nagai K. A New Synthetic-Aperture Focusing Method for Ultrasonic B-Scan Imaging by the Fourier Transform. Sonics and Ultrasonics, IEEE Transactions on. Jul; 1985 32(4):531-536.

13. Mayer K, Marklein R, Langenberg KJ, Kreutter T. Three-dimensional imaging system based on Fourier transform synthetic aperture focusing technique. Ultrasonics. Jul; 1990 28(4):241-255.

14. Busse LJ. Three-dimensional imaging using a frequency-domain synthetic aperture focusing technique. IEEE Trans Ultrason Ferroelectr Freq Control. Mar; 1992 39(2):174-179. [PubMed: 18263134]

15. Chang YF, Chern CC. Frequency-wavenumber migration of ultrasonic data. J Nondestruct Eval. 2000; 19(1):1-10.

16. Vray D, Haas C, Rastello T, Krueger M, Brusseau E, Schroeder K, Gimenez G, Ermert H. Synthetic aperture-based beam compression for intravascular ultrasound imaging. IEEE Trans Ultrason Ferroelectr Freq Control. Jan; 2001 48(1):189-201. [PubMed: 11367787]

17. Stepinski T. An Implementation of Synthetic Aperture Focusing Technique in Frequency Domain. IEEE Trans Ultrason Ferroelectr Freq Control. Jul; 2007 54(7):1399-1408. [PubMed: 17718329]

18. Hunter AJ, Drinkwater BW, Wilcox PD. The wavenumber algorithm for full-matrix imaging using an ultrasonic array. IEEE Trans Ultrason Ferroelectr Freq Control. Nov; 2008 55(11):2450-2462. [PubMed: 19049924]

19. Skjelvareid MH, Olofsson T, Birkelund Y, Larsen Y. Synthetic aperture focusing of ultrasonic data from multilayered media using an omega-k algorithm. IEEE Trans Ultrason Ferroelectr Freq Control. May; 2011 58(5):1037-1048. [PubMed: 21622059]

20. Cafforio C, Prati C, Rocca F. SAR data focusing using seismic migration techniques. IEEE Trans Aerosp Electron Syst. 1991; 27(2):194-207.

21. Milman AS. SAR imaging by w-k migration. Int J Remote Sens. 1993; 14(10):1965-1979.

22. Soumekh M. Reconnaissance with ultra wideband UHF synthetic aperture radar. IEEE Signal Process Mag. 1995; 12(4):21-40.

23. Lu JY. 2D and 3D high frame rate imaging with limited diffraction beams. IEEE Trans Ultrason Ferroelectr Freq Control. 1997; 44(4):839-856.

24. Lu JY. Experimental study of high frame rate imaging with limited diffraction beams. IEEE Trans Ultrason Ferroelectr Freq Control. 1998; 45(1):84-97. [PubMed: 18244161] 
25. Cheng J, Lu JY. Extended high-frame rate imaging method with limited-diffraction beams. IEEE Trans Ultrason Ferroelectr Freq Control. May; 2006 53(5):880-899. [PubMed: 16764444]

26. Kruizinga P, Mastik F, de Jong N, van der Steen AFW, van Soest G. Plane-wave ultrasound beamforming using a nonuniform fast fourier transform. IEEE Trans Ultrason Ferroelectr Freq Control. 2012; 59(12):2684-2691. [PubMed: 23221217]

27. Liu, DLD. Plane wave scanning reception and receiver. US Patent No. 6,685,641. Feb 3. 2004

28. Garcia D. BioméCardio website. 2012

29. Gazdag J, Sguazzero P. Migration of seismic data. Proc IEEE. 1984; 72(10):1302-1315.

30. Yilmaz, Ö. Seismic data analysis: processing, inversion, and interpretation of seismic data. Vol. I. SEG Books; 2001. Migration.

31. Schuck, A., Lange, G. Environmental Geology. Springer; Berlin Heidelberg: 2007. Seismic Methods; p. 337-402.

32. Culjat MO, Goldenberg D, Tewari P, Singh RS. A review of tissue substitutes for ultrasound imaging. Ultrasound Med Biol. Jun; 2010 36(6):861-873. [PubMed: 20510184]

33. Beatty PJ, Nishimura DG, Pauly JM. Rapid gridding reconstruction with a minimal oversampling ratio. IEEE Trans Med Imaging. Jun; 2005 24(6):799-808. [PubMed: 15959939]

34. Harlan W. Avoiding interpolation artifacts in Stolt migration. Stanford Exploration Project. 1982; 30:103-110.

35. Greengard L, Lee JY. Accelerating the nonuniform fast Fourier transform. SIAM Rev. 2004; 46(3): 443-454.

36. Song J, Liu QH, Torrione P, Collins L. Two-dimensional and three-dimensional NUFFT migration method for landmine detection using ground-penetrating radar. IEEE Trans Geosci Remote Sensing. 2006; 44(6):1462-1469.

37. van Wijk MC, Thijssen JM. Performance testing of medical ultrasound equipment: fundamental vs. harmonic mode. Ultrasonics. May; 2002 40(1-8):585-591. [PubMed: 12160006]

38. Yiu BYS, Tsang IKH, Yu ACH. GPU-based beamformer: Fast realization of plane wave compounding and synthetic aperture imaging. IEEE Trans Ultrason Ferroelectr Freq Control. 2011; 58(8):1698-1705. [PubMed: 21859591]

39. Hassanieh H, Indyk P, Katabi D, Price E. Nearly optimal sparse Fourier transform. 2012 Arxiv preprint arXiv:1201. 2501.

40. Schneider D. A faster fast fourier transform. IEEE Spectr. 2012; 49(3):12-13.

41. Schneider WA. Integral formulation for migration in two and three dimensions. Geophysics. 1978; 43(1):49-76.

42. Liu DL, Waag RC. Propagation and backpropagation for ultrasonic wavefront design. IEEE Trans Ultrason Ferroelectr Freq Control. 1997; 44(1):1-13. [PubMed: 18244096]

43. Jensen JA. Field: A program for simulating ultrasound systems. Med Biol Eng Comput. 1996; 34:351-353. [PubMed: 8945858]

44. Manes G, Tortoli P, Andreuccetti F, Avitabile G, Atzeni C. Synchronous dynamic focusing for ultrasound imaging. IEEE Trans Ultrason Ferroelectr Freq Control. 1988; 35(1):14-21. [PubMed: 18290124]

45. Wagner, N., Eldar, YC., Feuer, A., Danin, G., Friedman, Z. Xampling in ultrasound imaging. Proc. SPIE 7968, Medical Imaging 2011; Mar. 2011; p. 796818

46. Couture O, Fink M, Tanter M. Ultrasound contrast plane wave imaging. IEEE Trans Ultrason Ferroelectr Freq Control. 2012; 59(12):2676-2683. [PubMed: 23221216]

47. Shung KK, Zippuro M. Ultrasonic transducers and arrays. IEEE Eng Med Biol Mag. 1996; 15(6): 20-30.

48. Chen Q, Zagzebski JA. Simulation study of effects of speed of sound and attenuation on ultrasound lateral resolution. Ultrasound Med Biol. Oct; 2004 30(10):1297-1306. [PubMed: 15582229]

49. Gray SH, Etgen J, Dellinger J, Whitmore D. Seismic migration problems and solutions. Geophysics. Sep; 2001 66(5):1622-1640.

50. Anderson ME, Trahey GE. The direct estimation of sound speed using pulse-echo ultrasound. J Acoust Soc Am. Nov; 1998 104(5):3099-3106. [PubMed: 9821351] 
51. Pereira FR, Machado JC, Pereira WCA. Ultrasonic wave speed measurement using the time-delay profile of rf-backscattered signals: Simulation and experimental results. J Acoust Soc Am. Mar; 2002 111(3):1445-1453. [PubMed: 11931321]

52. Krucker JF, Fowlkes JB, Carson PL. Sound speed estimation using automatic ultrasound image registration. IEEE Trans Ultrason Ferroelectr Freq Control. Sep; 2004 51(9):1095-1106. [PubMed: 15478971]

53. Gazdag J. Wave equation migration with the phase-shift method. Geophysics. 1978; 43(7):13421351.

\section{Appendix: a simplified Matlab code for the Stolt's f-k migration with horizontal plane waves}

A simplified Matlab code is provided for Stolt's $f-k$ migration of RF signals with PWI. Note that this program is simplified and works with horizontal plane waves only. In addition, the number of options is limited. A complete protected code with in vitro examples is provided in the supplementary materials or can be downloaded from D. Garcia's webpage (www.biomecardio.com).

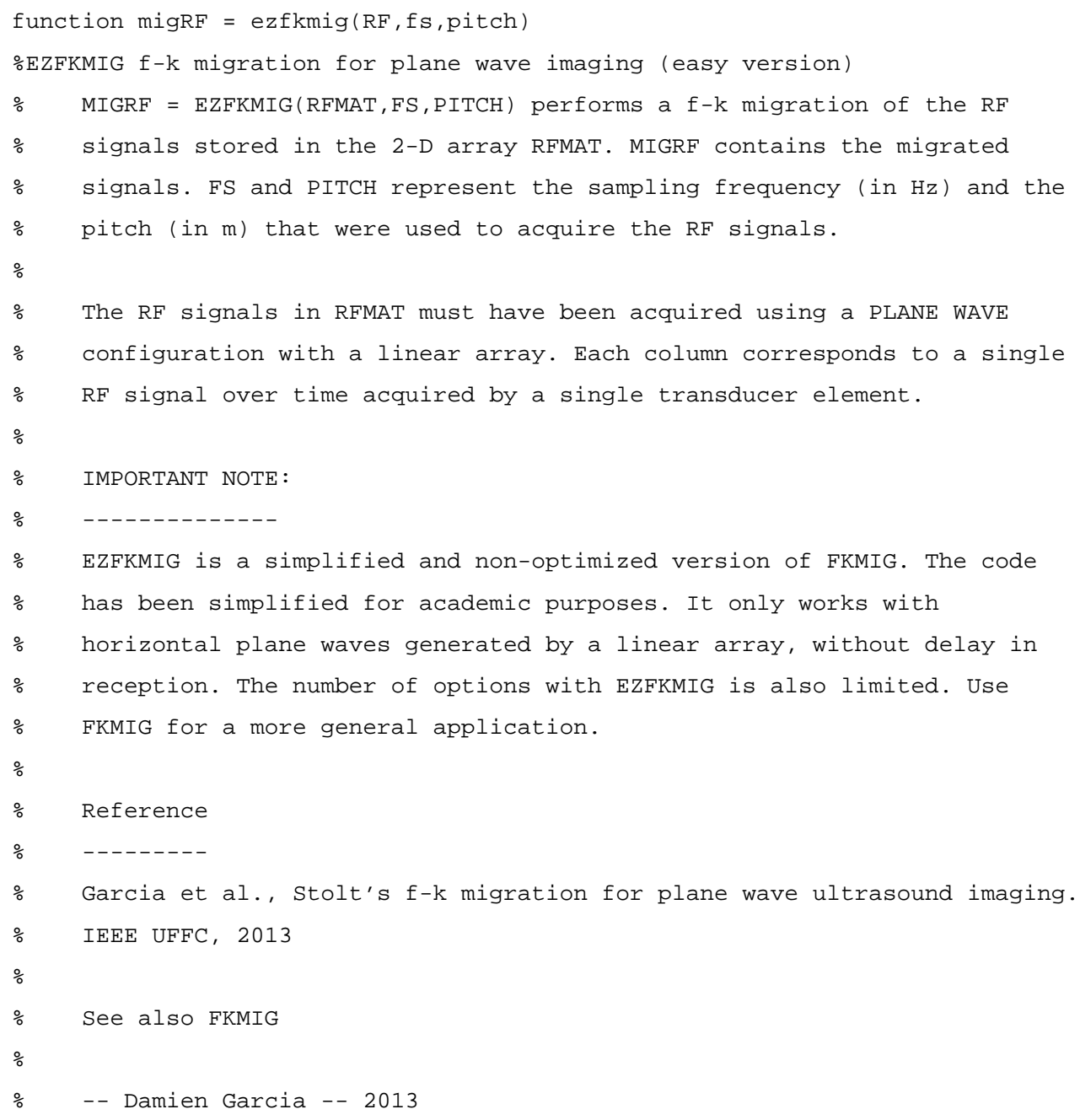




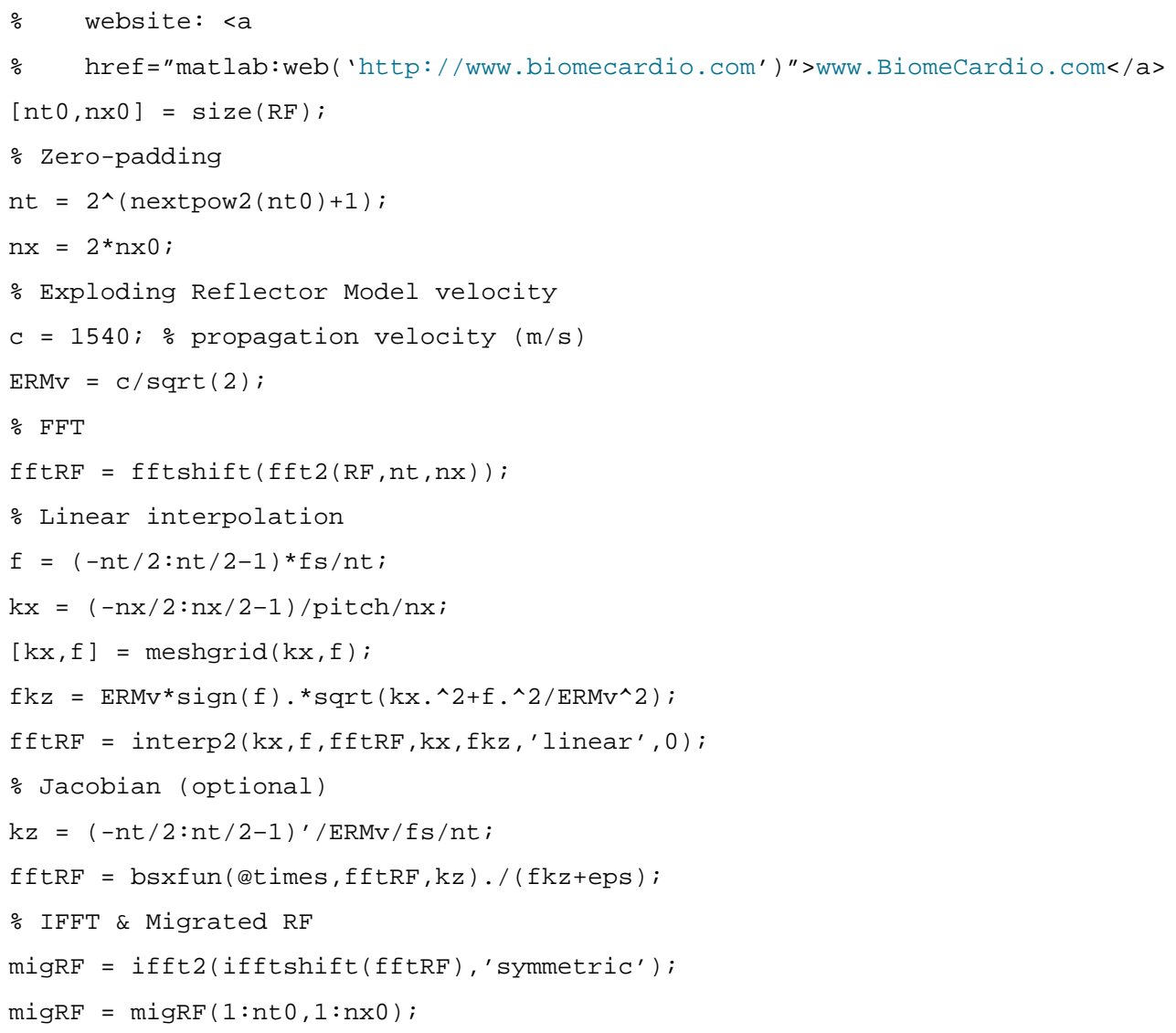

IEEE Trans Ultrason Ferroelectr Freq Control. Author manuscript; available in PMC 2014 March 31. 


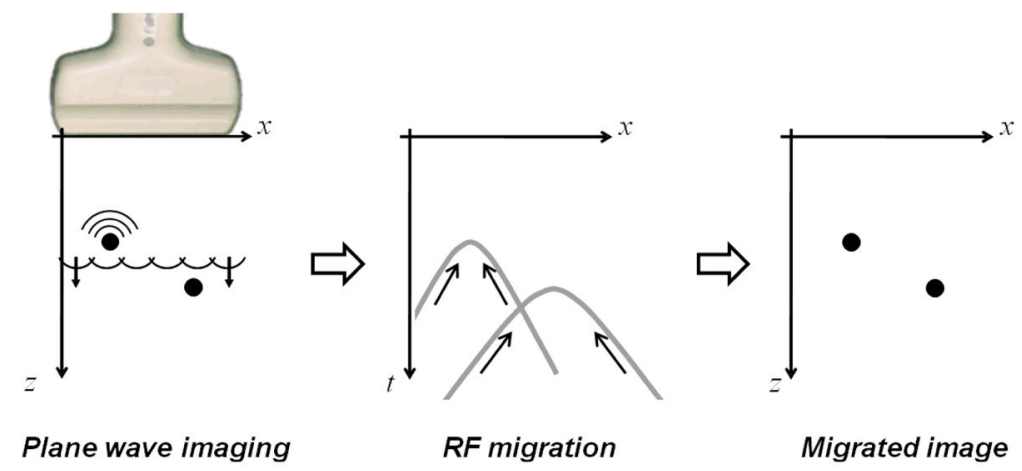

Figure 1. Migration process for plane wave imaging

As the scatterers are reached by a plane wave, they become secondary sources which emit upward spherical waves and generate diffraction hyperbolas in the RF backscattered signals. Migration allows one to recover the scatterers' positions and to reconstruct a B-mode image. 


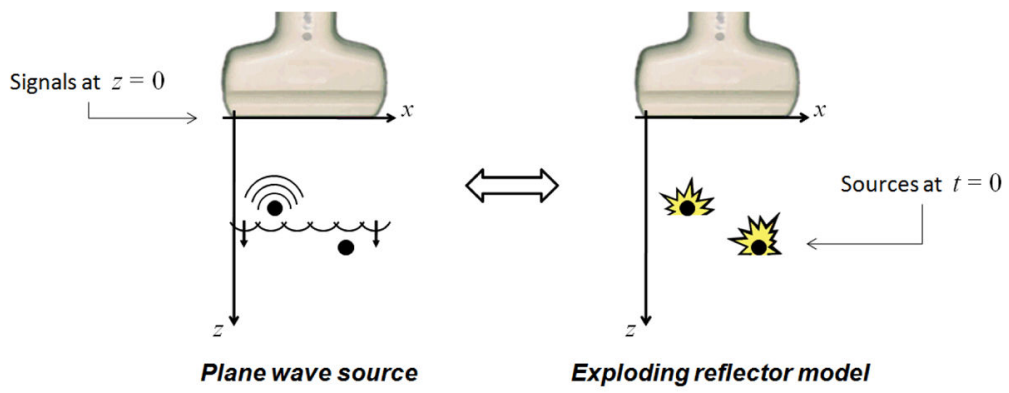

Figure 2. The exploding reflector model (ERM)

In the ERM, the scatterers are assumed to explode in concert, which simplifies the timereversal problem. To obtain the scatterers' positions, one must determine the wavefield at the time of explosion. In this study, the ERM, originally introduced for the seismic zero-offset configuration, is adapted to PWI. 


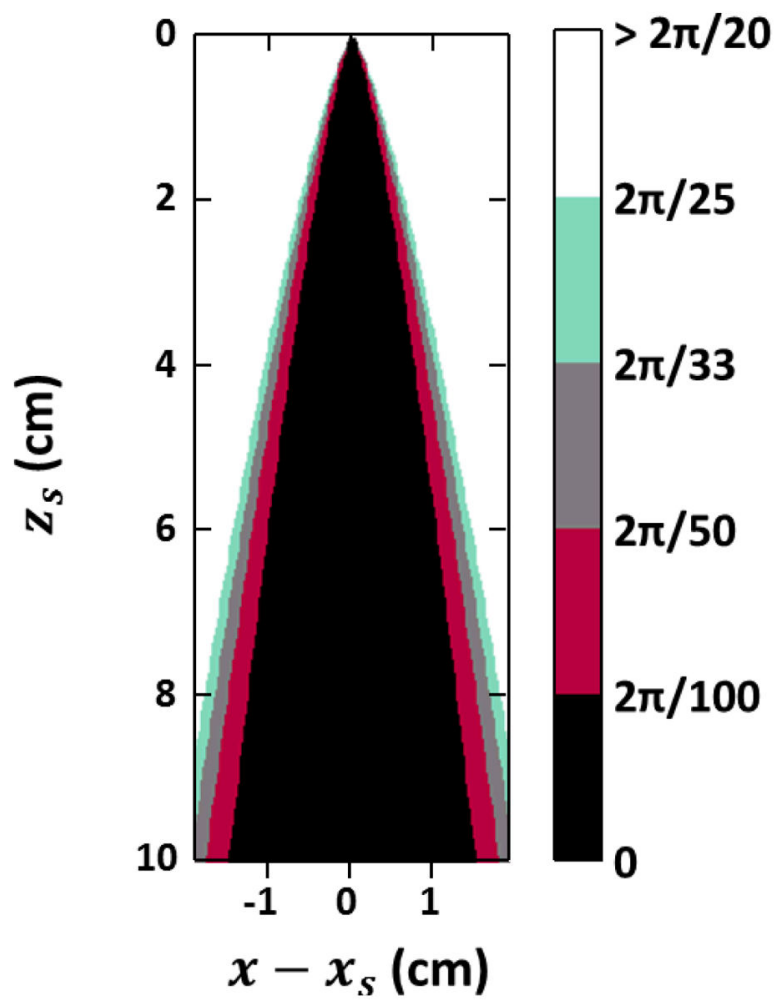

Figure 3. Phase errors induced to ERM modification

The travel-times of the classical ERM have been modified to make the ERM model adapted to plane wave imaging. The phase errors induced by these approximations depend upon the scatterer depth $\left(z_{S}\right)$ and on its position relative to the transducer element $\left(x-x_{S}\right)$. A $5 \mathrm{MHz}$ central frequency was used. 


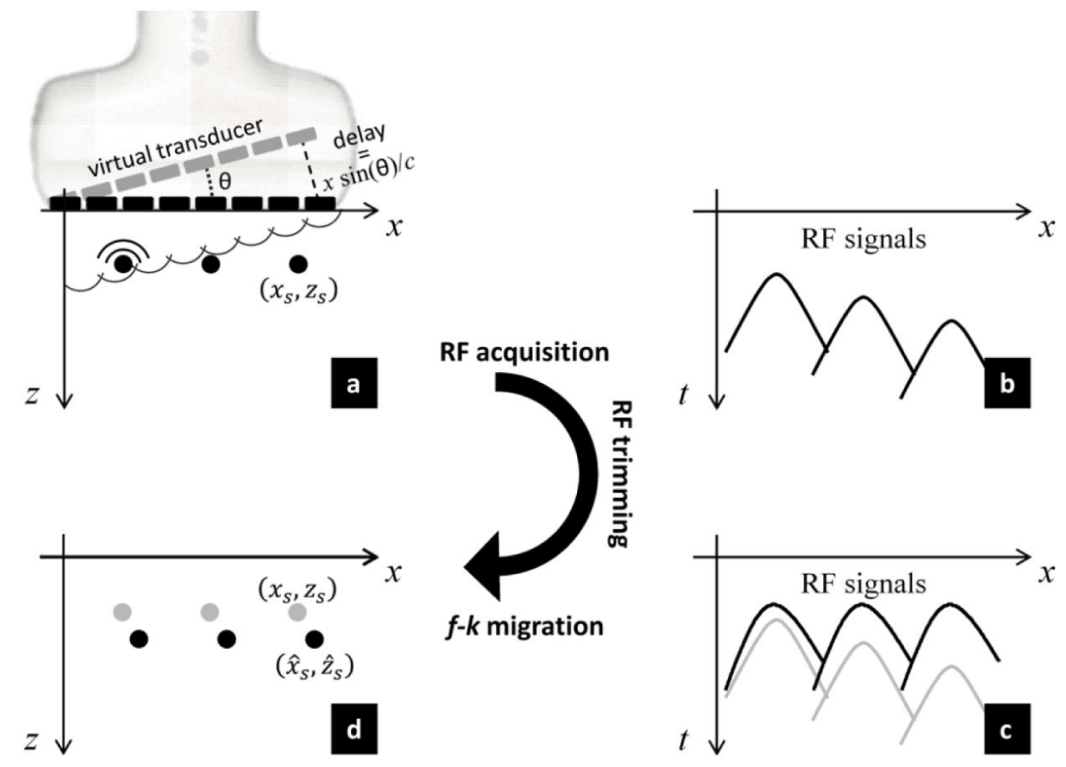

Figure 4. Adapting the $f$ - $k$ migration to tilted plane wave imaging

a) A slant plane wave is emitted. In this example, the leftmost scatterer is the first to perceive the planar wavefield. b) The resulting diffraction hyperbolas in the RF echoes are not aligned. c) Trimming the RF signals realigns the hyperbolas horizontally (from gray to black). d) A scatterer originally located at $\left(x_{S}, Z_{S}\right)$ will be moved to $\left(\hat{x}_{S}, \hat{z}_{S}\right)$ after the $f-k$ migration. 


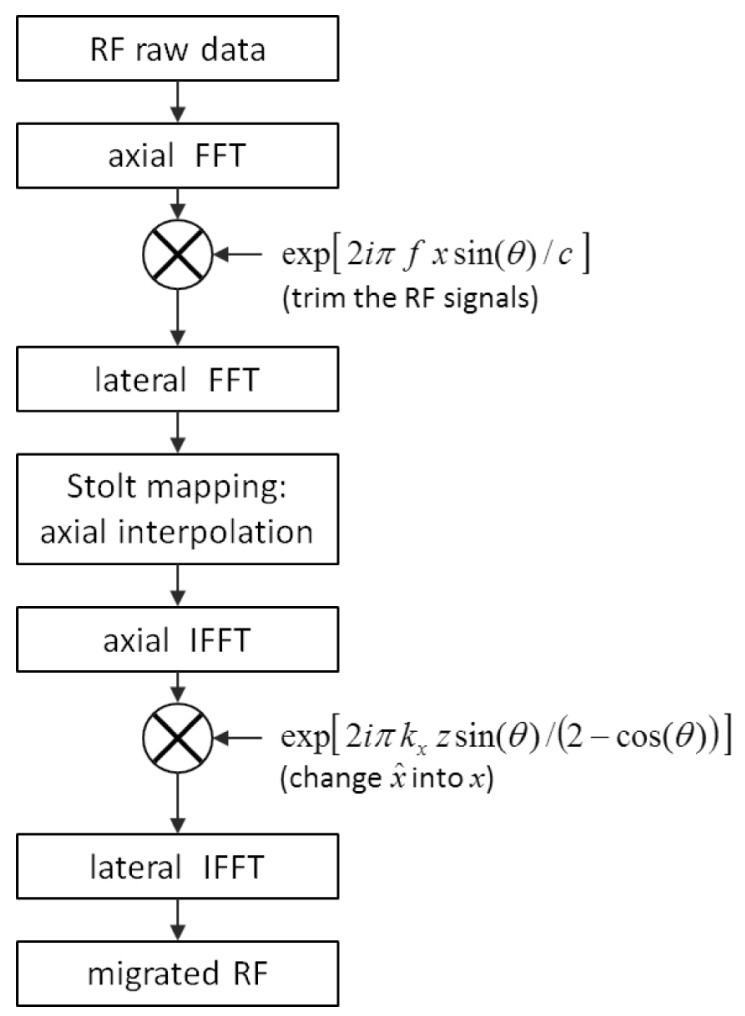

Figure 5. Diagram of the Stolt's $\boldsymbol{f}$ - $\boldsymbol{k}$ migration algorithm for plane wave imaging The proposed algorithm for migration of the RF signals works entirely in the Fourier domain. The coordinates $x$ and $z$ are defined as in Fig. 4. The parameters $k_{X}$ and $f$ stand for the spatial wavenumber and the temporal frequency, respectively. The wavefront angle $\theta$ is defined relative to the transducer (see Fig. 4). 

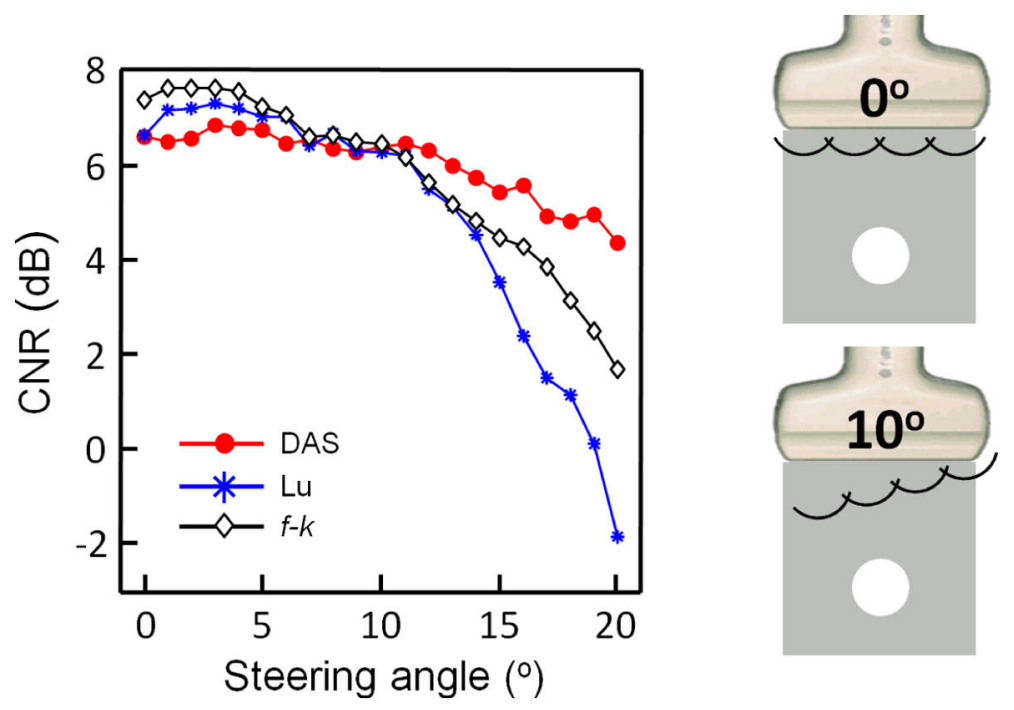

Figure 6. Effect of the steering angle on the contrast-to-noise ratio. In vitro results CNR for a $6 \mathrm{~mm}$-diameter $3 \mathrm{~cm}$-deep anechoic target (using a Gammex phantom and the Verasonics scanner). The CNR remained unchanged up to $10^{\circ}$ then decreased rapidly with angles $>10^{\circ}$, regardless of the migration method (DAS, Lu's method or $f-k$ migration). See also Fig. 6-bis in the Supplementary materials. 


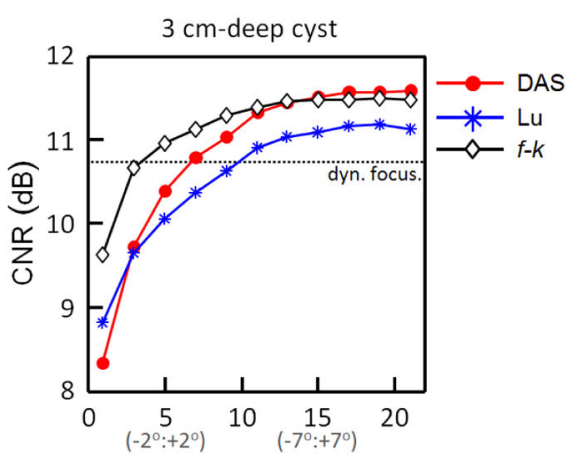

Number of angles
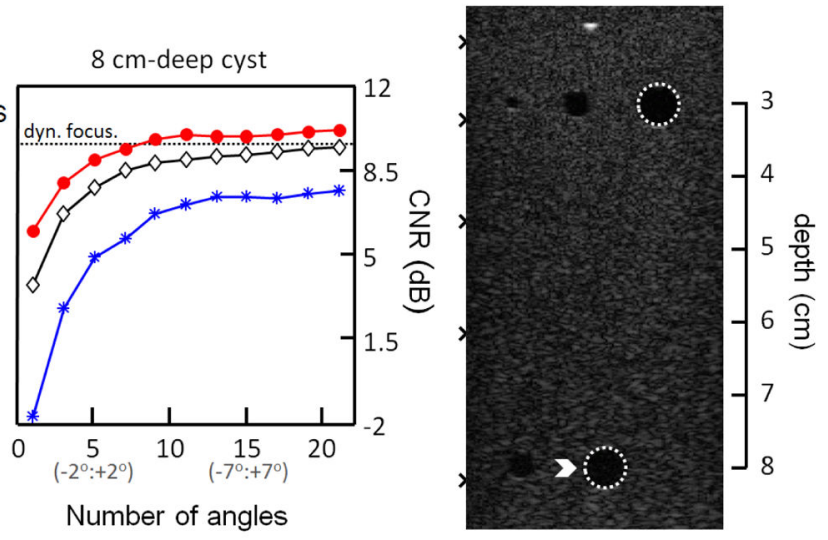

Figure 7. Effect of the number of compounding angles on the contrast-to-noise ratio. In vitro results

CNR for $6 \mathrm{~mm}$-diameter 3 and $8 \mathrm{~cm}$-deep anechoic targets (using a Gammex phantom and the Verasonics scanner). The targets of interest are delimited by dashed circles. Multi-angle compounding PWI provided CNR similar to those given by the dynamic focusing approach ("dyn. focus." in the figure, dashed horizontal lines). The locations of the foci are represented by the left markers in the rightmost image. The rightmost figure was obtained using $f$ - $k$ migration and seven compounding angles. See also Fig. 7-bis in the Supplementary materials. 

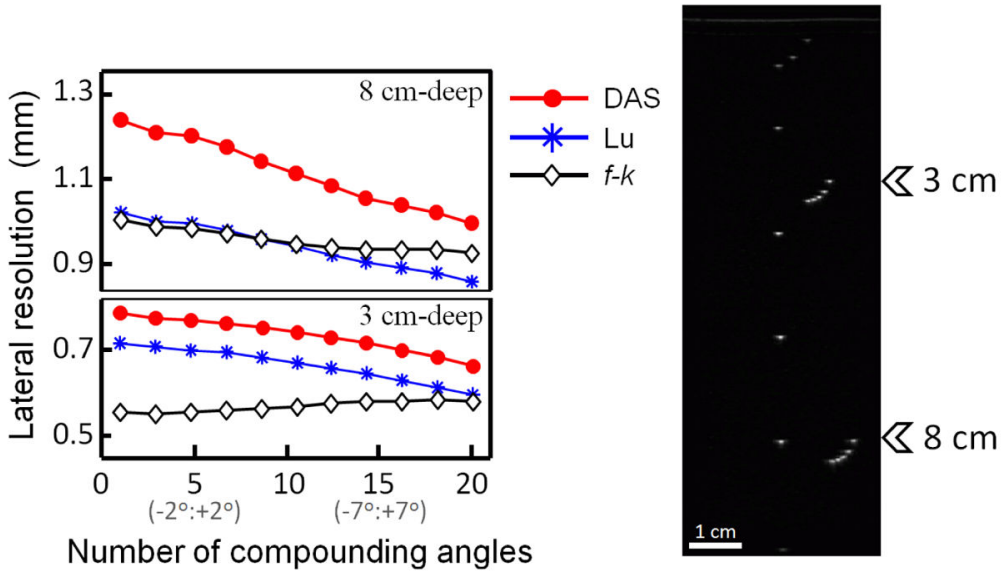

Figure 8. Effect of the number of compounding angles on the lateral resolution

Lateral resolution at 3 and $8 \mathrm{~cm}$ depth reached by multi-angle compounding PWI (using a Gammex phantom and the Verasonics scanner). The lateral resolution improved with increasing number of compounding angles. See also Fig. 9. 


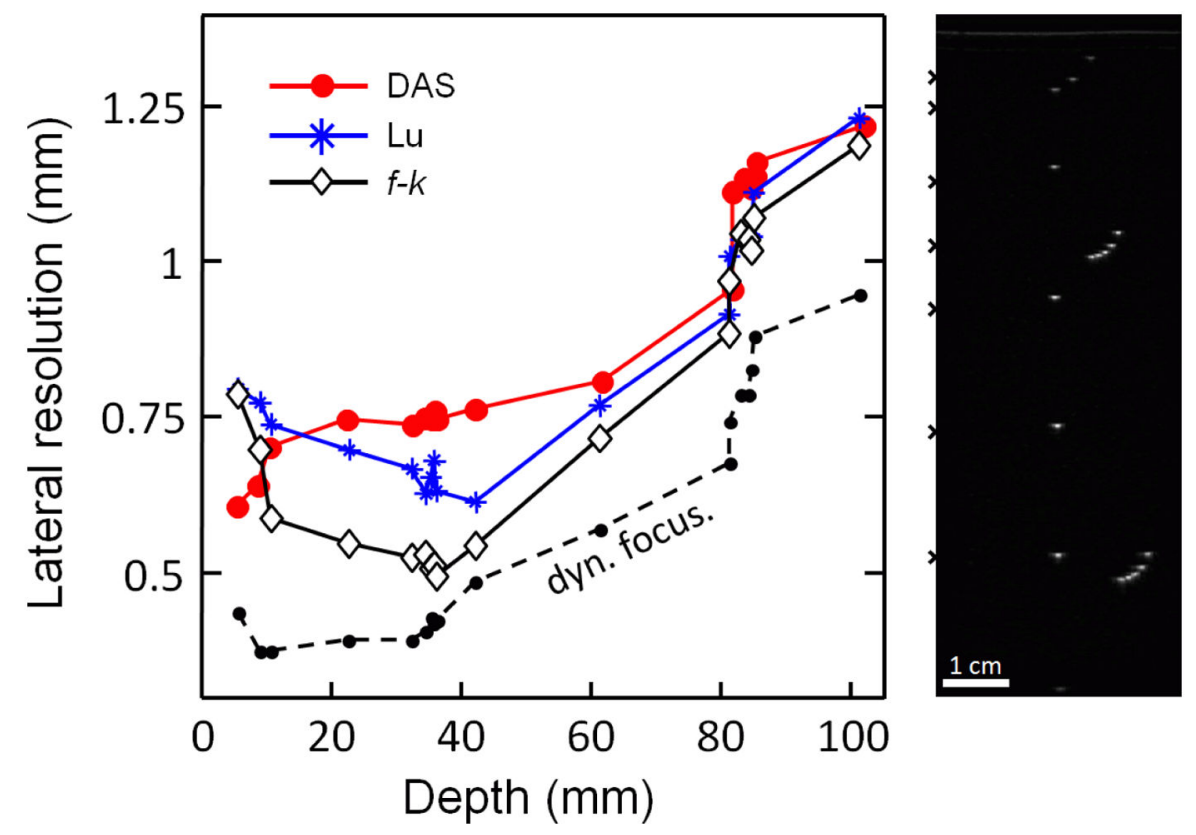

Figure 9. Effect of depth on the lateral resolution

Seven-angle compounded PWI images, reconstructed with the different migration methods (Lu's method, DAS or $f-k$ migration), were compared with dynamically focused images in a Gammex phantom using the Verasonics scanner. Only seven firing sequences were used to obtain a PWI image (see left grayscale image obtained with $f$ - $k$ migration) while 896 sequences were needed in the dynamic focusing mode ("dyn. focus." in the figure). The locations of the foci used in dynamic focusing are represented by the left markers in the rightmost image. 


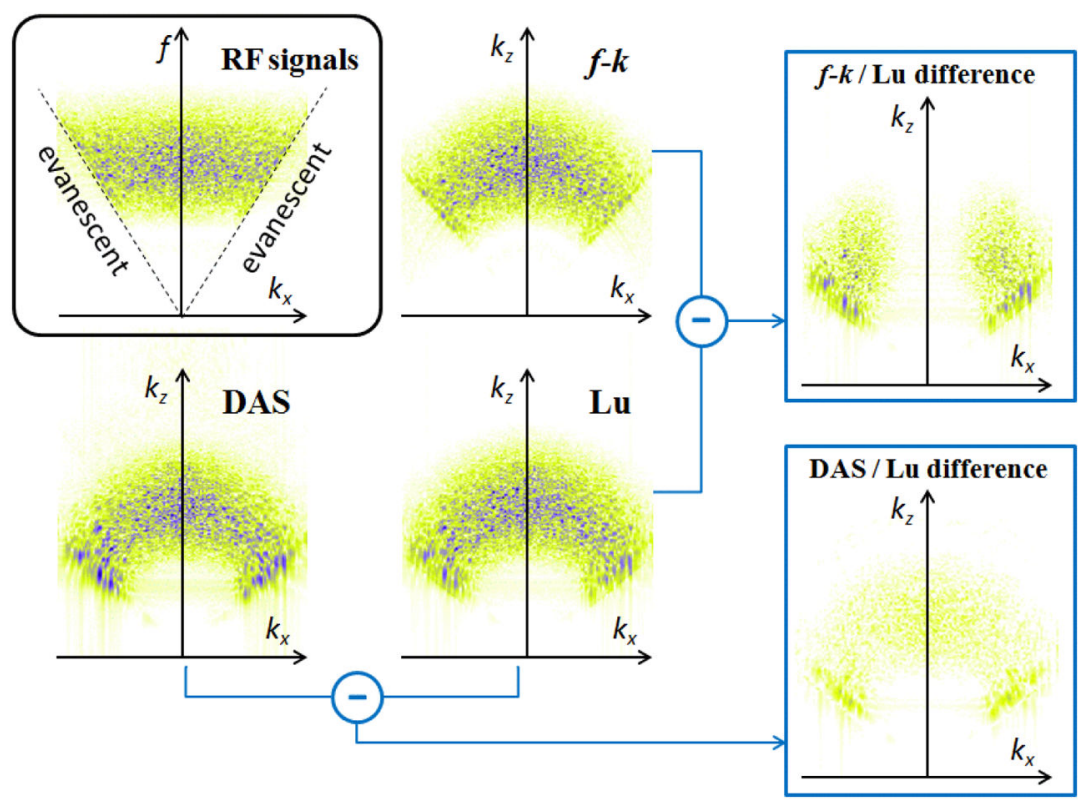

Figure 10. Spectral mapping

Spectral mapping of the migrated images using the three techniques (DAS, Lu's method, $f-k$ migration). The top left figure shows the spectrum of the raw RF signals before migration: $k_{X}$ stands for the spatial wavenumber related to $x$ and $f$ is the temporal frequency. The empty region, where $\left|f k_{X}\right|<c$, corresponds to the evanescent waves, which exhibit exponential decay in the very near-field. The rightmost figures represent the absolute differences of the Lu's and $f-k$ or DAS spectra. The RF signals were simulated using the freeware Field II [43] with randomly distributed scatterers. See also Fig. 10-bis in the Supplementary materials. 

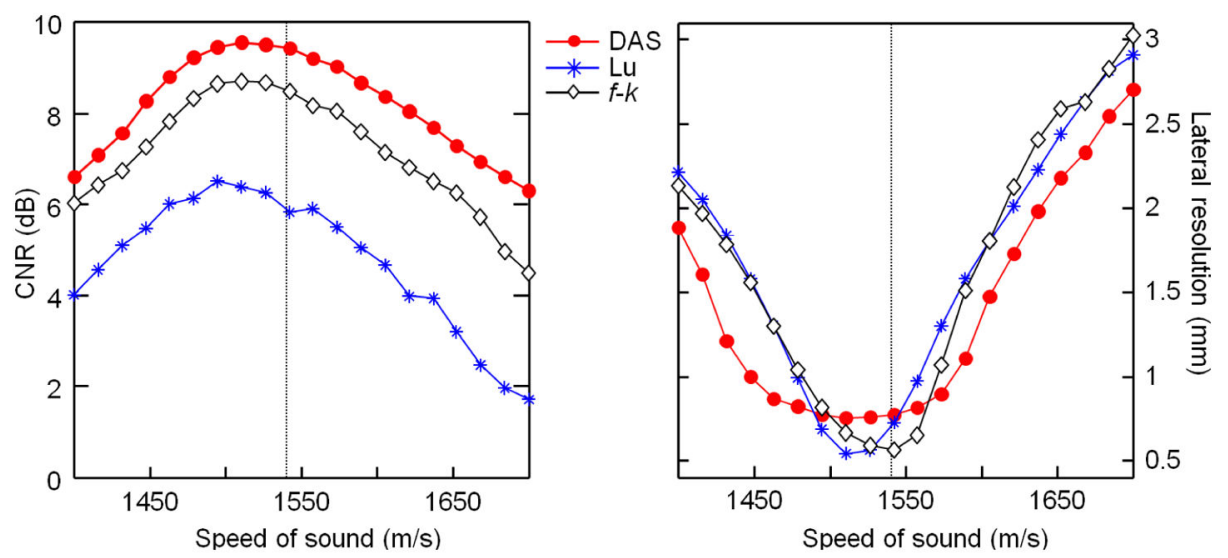

Figure 11. Effect of speed-of-sound image on image quality

Contrast-to-noise ratio (CNR) of the $8 \mathrm{~cm}$-deep cyst (left panel) and lateral resolution at 3 cm-depth (right panel) for different values of speed of sound (in vitro results, see also Fig. 7 and 8 ). The vertical dashed line represents the speed of sound value given by the documentation of the Gammex phantom. 